\title{
Intratumoral Ethyl Alcohol Injection for Devascularization of Hypervascular Intracranial Tumors
}

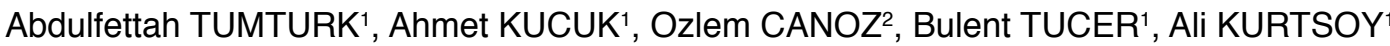 \\ ${ }^{1}$ Erciyes University Medical Faculty, Department of Neurosurgery, Kayseri, Turkey \\ ${ }^{2}$ Erciyes University Medical Faculty, Department of Pathology, Kayseri, Turkey
}

\section{ABSTRACT}

\begin{abstract}
AIM: Hypervascular intracranial tumors may cause serious bleeding in surgery. Though pre-operative endovascular embolization of tumor is a widely accepted method, sometimes despite embolization, an effective and safer intraoperative hemostatic technique is needed to prevent or at least decrease the massive bleeding from the tumors. The aim of this study was to investigate the effect of the ethyl alcohol (EA) injection in hypervascular tumors and find out whether it is likely to be an alternative method to prevent massive bleeding from tumor.
\end{abstract}

MATERIAL and METHODS: Fifty-five cases that had hypervascular intracranial tumor and underwent EA injections into their tumors were included in the study. A small amount (0.1-0.2 ml) of EA was used in every injection. Total EA amount differed from 1.2 to $18 \mathrm{ml}$ for each patient.

RESULTS: Most of the tumors were removed with less bleeding, because bleedings stopped or decreased during resection after EA injections. No serious complication that might be related to EA was observed.

CONCLUSION: EA injection into the tumor is an easy, cheap and less invasive method to obtain effective and safe tumor devascularization. It may be an alternative method for tumor devascularization when preoperative embolization cannot be performed due to any reason or severe bleeding despite embolization.

KEYWORDS: Intratumoral ethyl alcohol injection, Hypervascular tumor, Central nervous system, Intraoperative bleeding

\section{INTRODUCTION}

A safe and effective resection of intracranial tumors depends on meticulous homeostasis $(11,18,27)$. Brain tumors with rich vascular structures and deeply seated feeding arteries running at the opposite site of the surgical field may cause dangerous bleeding problems, which are likely to result in higher mortality and morbidity. The control of bleeding is usually difficult and the surgical duration is longer $(3,6,12)$. These unexpected and abrupt bleedings may not sometimes allow total excision of the tumor. Currently, the overall consensus within the field of neurosurgery is that preoperative tumor embolization is beneficial for reducing intraoperative blood loss in patients with vascular tumors $(6,12)$.
Pre-operative endovascular embolization of tumor feeders with various embolic agents is a widely accepted method $(3,6,18)$. The purpose of embolization is not only to occlude the vascular supply to the tumor, to achieve tumor necrosis and to decrease intraoperative blood loss, but also to maintain better visualization of surgical fields, which decreases the risk of adjacent tissue destruction and tumor recurrence (3). However, cases requiring emergent surgical intervention and tumors with feeders that also supply important normal anatomical structures may limit utilization of preoperative embolization $(6,18)$. Additionally, there may be some cases that undergo surgery in which massive bleeding during surgery is not expected. Consequently, reducing intratumoral blood flow or at least decreasing it to a more acceptable level 
prior to tumor resection is of vital importance. According to Bendszus et al. (1), embolization had an effect on blood loss only for patients with complete tumor devascularization. For this reason, an effective and safer intraoperative hemostatic approach is needed to improve the short and long-term prognosis of patients with intracranial tumors (27). Ethyl alcohol $(E A)$ has been extensively used as a successful sclerosing agent for interventional therapy of many disorders, including malignancies such as intraabdominal tumors and endocrine system tumors $(2,4,7-9,12,13,17,18)$. It is easily accessible, biocompatible, and less toxic if carefully used $(11,12,18,27)$.

In this study, we report our experience in fifty-five patients who underwent intratumoral injection of absolute EA during the resection of intracranial tumors.

\section{MATERIAL and METHODS}

This study was carried out with the ethics committee approval, decision number 2015/215 and date 17.04.2015, of Erciyes University, Medical Faculty. The patients were informed about the intratumoral EA injection, and informed consent was obtained before surgery.

Fifty-five of the 2567 cases (2.14\%) with intracranial tumors who were operated at Erciyes University, Medical Faculty, Neurosurgery Clinic between June 2004 and September 2013 were included in the study. Of the 55 cases, 30 were male and 25 were female. The mean age of the cases was 41.7 (ranging between 18 months and 67 years). All of the 55 cases had hypervascular intracranial tumors. Histopathological diagnoses of the intracranial lesions are given in Table I.

After surgical planning with a neuronavigation system (BrainLab VectorVision2, Munich, Germany), appropriate skin incisions and craniotomies were performed. $1 \mathrm{gr} / \mathrm{kg}$ of mannitol (20\%) was administered before dural incision for required cases. After the exposure of the tumor tissue, visible feeders were

Table I: Histopathological Diagnosis of the Fifty-Five Cases that Underwent Intraoperative Intratumoral EA Injection

\begin{tabular}{lcc}
\hline Histopathological Diagnoses & $\mathbf{n}$ & $\%$ \\
\hline Meningioma & 28 & 50.9 \\
\hline Glioblastoma Multiforme & 8 & 14.5 \\
\hline Medulloblastoma & 4 & 7.3 \\
\hline Astrocytoma & 4 & 7.3 \\
\hline Carcinoma Metastasis & 3 & 5.4 \\
\hline Cavernous Hemangioma & 2 & 3.6 \\
\hline Pituitary Adenoma & 2 & 3.6 \\
\hline Central Neurocytoma & 1 & 1.8 \\
\hline Glomus Jugulare Tumor & 1 & 1.8 \\
\hline Schwannoma & 1 & 1.8 \\
\hline Rhabdomyosarcoma Metastasis & 1 & 1.8 \\
\hline
\end{tabular}

coagulated. The tumor tissue was removed with the appropriate surgical technique. EA was used when excessive bleeding was observed during the tumor resection.

The margin of the injection side was determined using neuronavigation and restricted with a $0.5 \mathrm{~cm}$ distance to the tumor margin in encapsulated tumors and with a $1 \mathrm{~cm}$ distance to the tumor margin in un-encapsulated tumors. So, EA was not allowed to give harm to the adjacent neural tissues. B-mode ultrasonography was utilized not to inject EA into parent vessels.

Tuberculin injectors $(1 \mathrm{ml})$ with small diameter needles $(28$ gauge) were used for the dehydrated absolute EA (99.5\%) (Delta Kimya AŞ., İstanbul, Turkey) injection. Cotton pads with latex (latex obtained by cutting from surgical gloves) were used to prevent possible neural damage due to leakage of the EA from the injection site during the injection procedure (Figure 3). Additionally, aspiration and repeated injections of EA in small amounts $(0.1 \mathrm{ml}, 1 \mathrm{~cm}$ to the lateral border of the tumor and $0.2 \mathrm{ml}$, centrally) were used to avoid this complication. We performed negative aspiration before intratumoral EA injection to avoid injection into the parent vessels. Total amount of EA was in a range of $1.2-18 \mathrm{ml}$ (mean $7.3 \pm 4.9$ ) and varied according to the control of bleeding. Each injection was slowly performed within more than 60 seconds.

At the end of the injection, before it was slowly withdrawn, the needle was left in place for 30 seconds to avoid reflux of EA along the needle tract. Paleness in tumor color right after EA injection was a striking feature. Total resection of the tumor tissue followed tumor debulking with cavitron ultrasonic aspiration after devascularization of tumor tissue with EA injections. After meticulous homeostasis, surgical wounds were closed appropriately.

Magnetic resonance imaging (MRI) was performed on the first day of the post-operative period. One neuroradiologist and two neurosurgeons evaluated the surgical area in terms of hemorrhage, residual tumor and brain edema.

We compared the pre-operative and post-operative hemoglobin values, suction trap volume, intraoperative and postoperative systemic blood pressure (from the point of hypotensive episodes due to blood loss), the number of patients who underwent blood transfusion and the duration between dural opening and closure of the patients whose tumors were administered alcohol with the results of 40 randomly and retrospectively chosen patients, most of whom had meningiomas and were operated without using alcohol at the same period.

The independent samples $t$ test was used for comparisons between groups. $p<0.05$ was considered statistically significant.

\section{RESULTS}

In all of the cases, marked whitening of tumor tissue at the injection sites and regional devascularization were observed. On the contrary, active bleeding was continuing at the tumor regions that did not undergo EA injection. These areas also became pale after EA injections and bleeding stopped right 
after the injections. Tumor tissues that received EA injection became softer and brittle in 2-4 minutes. These new features of the tumors allowed easier and more rapid resection of the tumors. We did not observe any significant difference in the pre-operative and post-operative hemoglobin values between the control group and the patients whose tumors were administered alcohol. Moreover, there was no significant difference between the groups from the point of blood transfusion frequency, blood volume in the suction trap and duration of the surgery. No postoperative hypotensive episodes secondary to blood loss were recorded for either group. Length of hospital stay was also equal between the two groups,. The pre-operative and post-operative hemoglobin values, intraoperative and postoperative systemic blood pressure values, and surgical duration between dural opening and closure are given in Table II.

Since there was decreased bleeding, duration of homeostasis was shorter and the total surgical time was decreased. The postoperative neurological and radiological findings showed no EA-related complications (Figures 1A-D; 2A-C; 3). There was no permanent neurological damage.

Table II: The Pre-Operative and Post-Operative Hemoglobin Values, Blood Volume in Suction Trap, Surgical Duration Between Dural Opening and Closure and Intraoperative and Postoperative Systemic Blood Pressure Values of the Cases

\begin{tabular}{lccc}
\hline & EA used $(\mathbf{n = 5 5 )}$ & EA unused $(\mathbf{n = 4 0 )}$ & $\mathbf{p}$ value \\
\hline Preoperative Hb (gr/dl) & $14.1 \pm 2.0$ & $13.5 \pm 1.8$ & 0.136 \\
\hline Postoperative Hb (gr/dl) & $12.6 \pm 1.9$ & $11.9 \pm 2.0$ & 0.086 \\
\hline Blood in Suction Trap (ml) & $415 \pm 169$ & $375 \pm 158$ & 0.245 \\
\hline Duration of Surgery (minute) & $68 \pm 16$ & $69 \pm 17$ & 0.770 \\
\hline Intraoperative S/D BP (mmHg) & $105 \pm 15 / 67 \pm 13$ & $100 \pm 14 / 66 \pm 12$ & $0.112 / 0.703$ \\
\hline Postoperative S/D BP (mmHg) & $140 \pm 18 / 77 \pm 14$ & $135 \pm 17 / 74 \pm 13$ & $0.175 / 0.290$ \\
\hline
\end{tabular}

S/D BP: Systolic/Diastolic Blood Pressure, Hb: Hemoglobin.
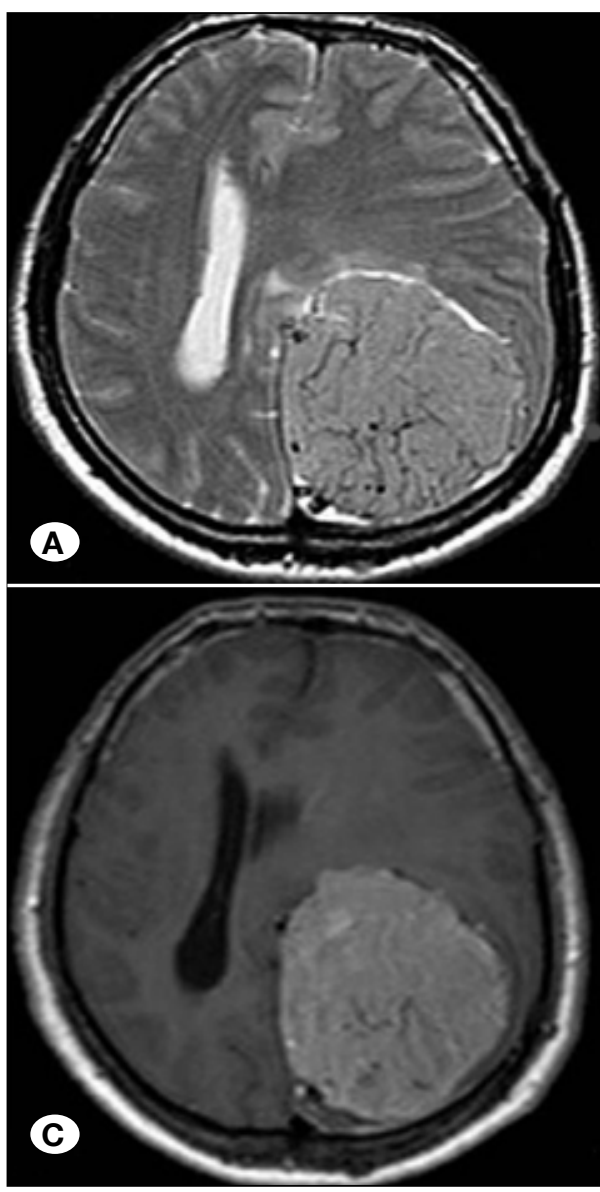

Figure 1: T2W axial (A) and coronal (B) MRI scans show a mass lesion, which has a shift effect on the left parietooccipital region and rich vascular structures and feeders of the mass, as well. Postcontrast T1W axial (C) and sagittal (D) images show an intensive contrast enhancement and also rich vascular structures in the mass. 


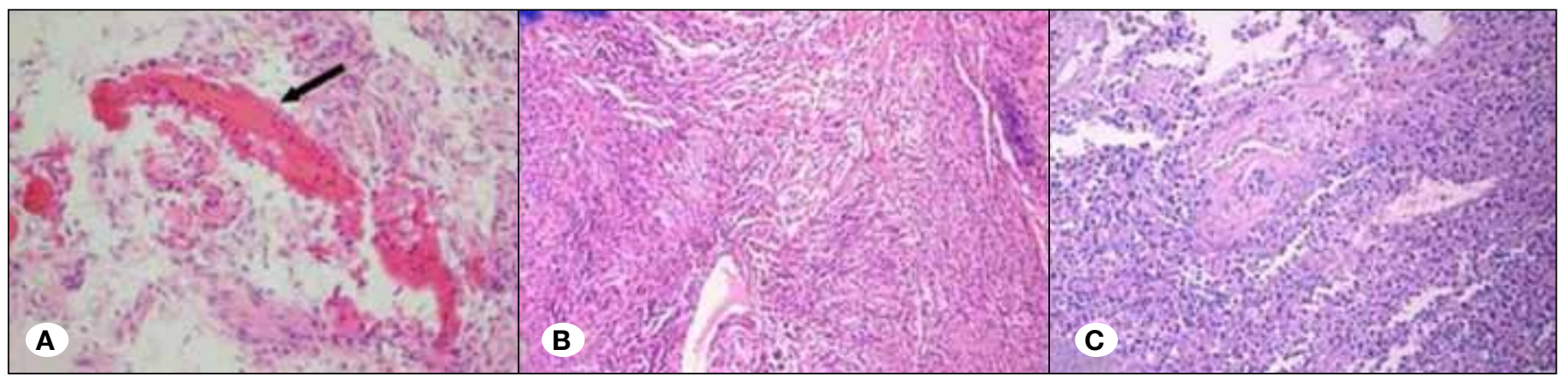

Figure 2: Histopathological findings show the damage of the endothelial cells and congestion with consequent thrombosis (arrow) of the small vessels in meningioma (A). Coagulation necrosis is observed around the affected vessels in tumor tissues. Cells appear to be silhouettes (middle-upper portion of the image) $(H / E ; x 40)(B)$. Two vessels were with damaged endothelium. Around these vessels, neutrophil leukocytes were monitored (H/E; x40) (C).

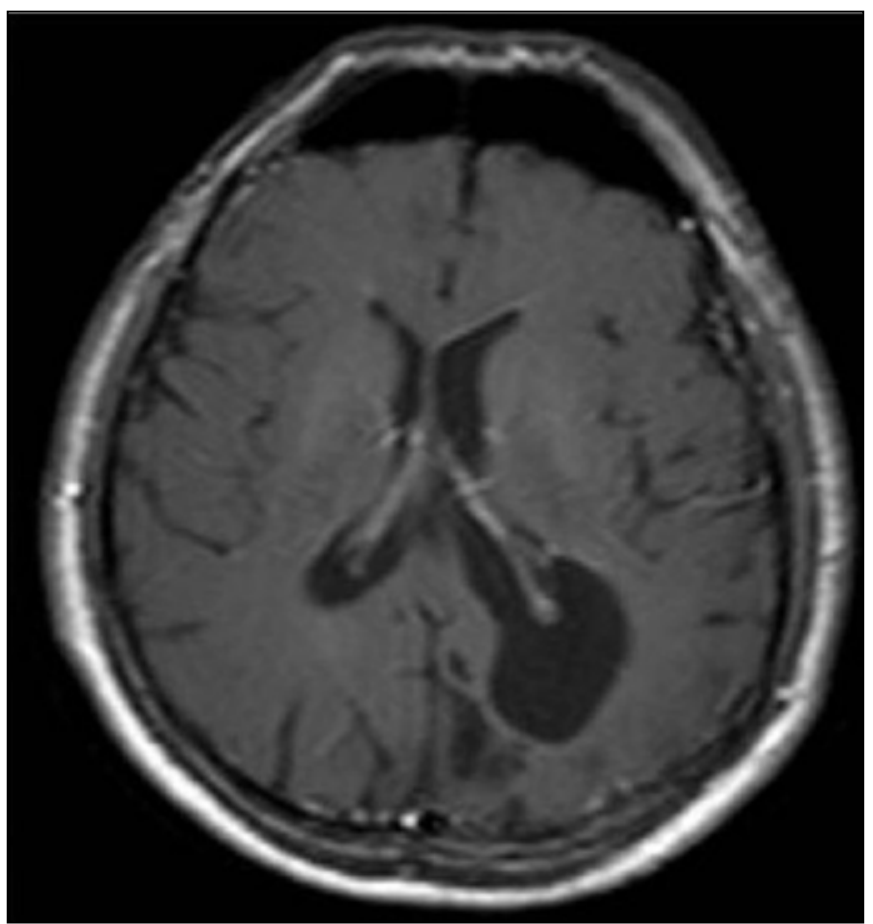

Figure 3: Axial MRI scan of the case 8 months after surgery.

\section{DISCUSSION}

Reducing tumoral blood flow before surgery is of vital importance in preventing dangerous bleedings from tumors that have rich vasculature. Endovascular embolization of tumor feeders 24-72 hours prior to surgery with interventional radiological methods is a well-known and safe method for this purpose $(3,18,27)$. Various agents such as fibrin glue, polyvinyl alcohol particles, gelfoam, cyanoacrylate glue etc. have been used for tumor feeders' embolization (6). However, factors such as arteries that supply both the tumor and the normal brain $(6,18)$, technical obstacles that prevent embolization, cases that require emergent surgeries, and tumors that are completely intra-axial may limit deployment of this technique. Some other techniques such as intraoperative coagulation of visible tumor feeders may help to prevent massive intraoperative bleeding from the tumor. Yet, there are some tumors like meningiomas and hemangiopericytomas the feeders of which either are not clearly exposed or are hidden at the opposite side of the surgical exposure.

EA may be used intravascularly to embolize vascular $(7,8$, $28,29)$ and neoplastic $(4,5)$ structures. In their experimental study, Ellman et al. (5) have demonstrated that acute arterial occlusion occurred with slow EA injection via intravascular rates due to embolization by damaged blood components. In addition, when administered percutaneously, EA is an agent that effectively leads to devascularization and sclerosis of endocrine tissues $(16,21,24)$, intra-abdominal tumors $(17,23)$ and vascular lesions $(28,29)$. Trans-cutaneous and intralesional injections of EA have been widely used for devascularization of tumors such as hepatocellular carcinoma (26), benign thyroid nodules (30) and thymic cysts (9). Another field for use of EA is neuro-oncology. It has been used after pituitary surgery for residual tumors (15). Additionally, EA has been injected into the hypophysis gland for neuro-adenolysis to reduce intractable pain $(22,25)$.

Lonser et al. (18) have used percutaneous EA infusions for vertebral hemangiomas and intraoperative direct injections for other lesions, and achieved reduced bleeding during excision of these pathologies. Furthermore, they used this technique successfully for a case that had a massive bleeding during the surgery in spite of preoperative embolization. Tumoral devascularization with direct EA perfusion during surgery seems to be a feasible method for cases that have tortuous vasculature preventing preoperative embolization, or that have common feeders that supply both the tumor tissue and the normal brain structures. Likewise, cases that cannot undergo preoperative embolization for any reason, or cases that require emergent surgery, are excellent candidates for intraoperative EA injection. Isaka et al. (12) have described a surgical technique consisting of a combination of intraoperative ultrasonographically guided intratumoral EA injection for highly vascularized brain tumor.

The mechanism of action of lesional EA injection may be due to coagulative necrosis and vascular thrombosis with hemorrhagic infarction $(4,5,10,14)$. In support of this, in our study, we observed damage of the endothelial cells, 
congestion, with consequent thrombosis of the small vessels and coagulative necrosis around the affected vessels in histopathology.

Mesiwala et al. (19) have studied the contact effects of EA on neural tissues. They have demonstrated both in vivo mesothelial effects of EA leading to stromal, neuronal, astrocytic and glial degeneration, and evident hydrogen peroxide-like antitumoral effects. Due to the evident neurotoxic effects of EA, direct contact of this agent with the neural tissue must be avoided. Using cotton pads with latex on the normal neural tissue, small caliber needles and meticulous aspiration of the leaking alcohol during the application may help to prevent this complication. In the literature, various amounts of EA (3 to $27 \mathrm{ml}$ ) were used in intracranial tumors by authors and the complications of the EA injections are rarely reported and are mainly thought to be dose-depended. Isaka et al. (11) reported such complications of intratumoral EA injection as nausea and dizziness lasting for about 3 days in a patient with giant skull base metastasis, which is attributed to the mild alcohol intoxication. Likewise, symptoms that can be attributed to mild transient alcohol intoxication were observed in 9 cases who were administered more than $10 \mathrm{ml}$ alcohol.

Why we correlated the complaints of vomiting and dizziness we observed in these cases with alcohol was the fact that we observed these complaints only in the cases who were administered over $10 \mathrm{ml}$ alcohol. We thought that the reason of this situation might be the low amount of diluted alcohol (due to SF administered to the tumor area during bipolar coagulation and cavitron ultrasonic surgical aspirator (CUSA) use) that enters into the systemic circulation by being absorbed from the tumor-area, which is related to the continuing intratumoral blood circulation in spite of alcohol injection. However, in this study, a separate investigation was not carried out on these possible complications.

In the light of literature, what we have found out in this retrospective study was that alcohol injection was a possible procedure to be used only by keeping tightly to the criteria of EA injection, preventing direct contact of alcohol with neural tissue and not injecting into the parent vessels; however, we are of the opinion that the risk in the cases in whom tumor boundaries and parent vessels could not be determined (such as central neurocytoma) precisely is high.

Okada et al. (20) have reported a serious complication, a bilateral thalamic infarct, in a case they operated on in two stages. They said that they believed the injection of ethanol near the draining vessel caused intraluminal coagulation and embolized the internal cerebral veins and the great vein of Galen. However, the patient loss of these authors may be attributed to the hemorrhage seen on postoperative MRI, as well. We used intraoperative ultrasonography to avoid injection into parent vessels and did not meet that kind of complication. Although it is stated that it was safer and easier to use in encapsulated tumors but dangerous in unencapsulated tumors, we used EA injections in deeply seated and un-encapsulated tumors such as gliomas and none of the cases have demonstrated any serious side effects that may be attributed to EA.
The criteria to determine the amount of the alcohol were not only to obtain an acceptable bleeding level to perform safe tumor removal, but to prevent from an excessive blood loss that may be able to give rise to the cancellation of the surgery or incomplete resection, as well. Alcohol injection was ended when these two aims were achieved.

As well as the factors such as tumor size, contrast level and surrounding tumoral edema, the most common factors in choosing the patients was excessive hemorrhage that we had difficulty in controlling with bipolar coagulation during the surgery.

We did not observe any significant difference between the pre-operative and post-operative hemoglobin values and the blood volume in suction trap of the randomly and retrospectively chosen operated patients most of whom had meningiomas without using alcohol at the same period and of the patients whose tumors were administered alcohol. Moreover, there was no significant difference between the groups from the point of blood transfusion frequency and duration of the operations, which led us to think that all these outcomes were due to EA use.

\section{CONCLUSION}

Direct EA injection to the tumor tissue is an easy, cheap and less invasive method that can be used for almost all of the cases with intraoperative excessive bleeding. Consecutive injections of small amounts of EA result in fast, effective and safe tumor devascularization. Careful application of this technique will decrease tumor bleeding and lead to safer and easier tumor resections.

Clean microsurgery and preoperative embolization are the mainstay of the hypervascular intracranial tumor surgery. In spite of the fact that there are some complications and difficulties to use this technique in an intra-axially seated tumor, intratumoral EA injections may be an alternative to preoperative embolization when preoperative embolization cannot be performed due to any reason and it can be used in the patients who have serious bleeding despite the embolization.

\section{REFERENCES}

1. Bendszus M, Rao G, Burger R, Schaller C, Scheinemann K, Warmuth-Metz M, Hofmann E, Schramm J, Roosen K, Solymosi $\mathrm{L}:$ Is there a benefit of preoperative meningioma embolization? Neurosurgery 47:1306-1312, 2000

2. Crescenzi A, Papini E, Pacella CM, Rinaldi R, Panunzi C, Petrucci L, Fabbrini R, Bizzarri GC, Anelli V, Nardi F, Marinozzi V: Morphological changes in a hyper functioning thyroid adenoma after percutaneous ethanol injection: Histological, enzymatic and sub-microscopical alterations. J Endocrinol Invest 19:371-376, 1996

3. Duffis EJ, Gandhi CD, Prestigiacomo CJ: Head, neck, and brain tumor embolization guidelines. J Neuro Intervent Surg 4:251-255, 2012

4. Ellman BA, Parkhill BJ, Curry TS III, Marcus PB, Peters PC: Ablation of renal tumors with absolute ethanol: $A$ new technique. Radiology 141: 619-626, 1981 
5. Ellman BA, Parkhill BJ, Marcus PB, Curry TS III, Peters PC: Renal ablation with absolute ethanol: Mechanism of action. Invest Radiol 19:416-423, 1984

6. Gupta AK, Purkayastha S, Bodhey NK, Kapilamoorthy TR, Kesavadas C: Preoperative embolization of hypervascular head and neck tumours. Australian Radiol 51:446-452, 2007

7. Heiss JD, Doppman JL, Oldfield EH: Brief report: Relief of spinal cord compression from vertebral hemangioma by intralesional injection of absolute ethanol. N Eng J Med 331: 508-511, 1994

8. Heiss JD, Doppman JL, Oldfield EH: Treatment of vertebral hemangioma by intralesional injection of absolute ethanol. N Eng J Med (letter) 334:1340, 1996

9. Hirano $Y$, Shimada T, Kinoshita Y, Murakami Y, Kobayashi K, Yoshitomi H, Kitamura J, Sano K, Tanabe K, Ishibashi Y, Murakami $\mathrm{R}$ : Ethanol sclerosis: One of the best treatments for thymic cyst in very elderly patients? Intern Med 36: 716-719, 1997

10. Horowitz M, Whisnant RE, Jungreis C, Snyderman C, Levy El, Kassam A: Temporary balloon occlusion and ethanol injection for preoperative embolization of carotid-body tumor. Ear Nose Throat J 81:536-538, 2002

11. Isaka T, Nakagawa $H$, Suzuki T, Yamada J, Wada K, Kadota T: Successful removal of a giant skull base metastasis from hepatocellular carcinoma after direct ethanol injection: Case report. Skull Base Surg 10:81-86, 2000

12. Isaka T, Nakagawa H, Yamada J, Suzuki T, Wada K: Intraoperative ultrasonographically guided direct ethanol injection for a brain metastasis from renal cell carcinoma: Case report. Neurosurg Rev 24:123-126, 2001

13. Jungreis JA: Skull-base tumors: $E T O H$ embolization of the cavernous carotid artery. Radiology 181:741-743, 1991

14. Konya A, Van Pelt CS, Wright KC: Ethiodized oil-ethanol capillary embolization in rabbit kidneys: Temporal histopathologic findings. Radiology 232: 147-153, 2004

15. Laws ER Jr: Transsphenoidal approach to pituitary tumors. In: Schmidek HH, Sweet WH (eds), Pituitary Tumors (Operative Neurosurgical Techniques: Indications, Methods and Results) Philadelphia: W B Saunders, 1995: 283-292

16. Lippi F, Ferrari C, Manetti L, Rago T, Santini F, Monzani F, Belitti P, Papini E, Busnardo B, Angelini F, Pinchera A: Treatment of solitary autonomous thyroid nodules by percutaneous ethanol injection: Results of an Italian multicenter study. J Clin Endocrinol Metab 81:3261-3264, 1996

17. Livarghi T, Festi D, Monti F, Salmi A, Vettori C: US-guided percutaneous alcohol injection of small hepatic and abdominal tumors. Radiology 161:309-312, 1986
18. Lonser RR, Heiss JD, Oldfield EH: Tumor devascularization by intratumoral ethanol injection during surgery. J Neurosurg 88: 923-924, 1998

19. Mesiwala AH, Farrel L, Santiago P, Ghatan S, Silbergerd DL: The effect of hydrogen peroxide on brain and brain tumors. Surg Neurol 59: 398-407, 2003

20. Okada Y, Taniguchi M, Takahashi H: Intratumoral ethanol injection. (Letter). J Neurosurg 91:521-523, 1999

21. Papini E, Panunzi C, Pacella CM, Bizzari G, Fabbrini R, Petrucci L, Pisicchio G, Nardi F: Percutaneous ultrasound-guided ethano injection: A new treatment of toxic autonomously functioning thyroid nodules? J Clin Endocrinol Metab 76:411-416, 1993

22. Roberts MT, Henderson RS: Pituitary fossa injection with alcohol for widespread cancer pain. N Z Med J 93:1-3, 1981

23. Sakamoto M, Hirohashi S: Natural history and prognosis of adenomatous hyperplasia and early hepatocellular carcinoma: Multi-institutional analysis of 53 nodules followed up for more than six months and 141 patients with single early hepatocellular carcinoma treated by surgical resection or percutaneous $\mathrm{ETOH}$ injection. Jpn J Clin Oncol 28: 604-608, 1998

24. Solbiati L, Giangrande A, De Pra L, Bellotti E, Cantu P, Ravetto C: Percutaneous ethanol injection of parathyroid tumors under US guidance: Treatment for secondary hyperthyroidism. Radiology 155: 607-610, 1985

25. Takeda F, Uki J, Fujii T, Kitani Y, Fujita T: Pituitary neuroadenolysis to relieve cancer pain-observation of spread of ETOH instilled into the sella turcica and subsequent changes of the hypothalamopituitary axis at autopsy. Neurol Med Chir 23:50-54, 1983

26. Tanaka K, Okazaki H, Nakamura S, Endo O, Inoue S, Takamura Y, Sugiyama M, Ohaki Y: Hepatocellular carcinoma: Treatment with a combination therapy of transcatheter arterial embolization and percutaneous ethanol injection. Radiology 179:713-717, 1991

27. Wang G, Li J, Zhang H, Wu Y, Lv Y, Zhu J: Anhydrous ethanol reduces intraoperative hemorrhage of intracranial highly vascularized large meningiomas. Chinese-German J Clin Oncol 9:344348, 2010

28. Yakes WF, Haas DK, Parker SH, Gibson MD, Hopper KD, Mulligan JS, Pevsner PH, Johns JC Jr, Carter TE: Symptomatic vascular malformation: Ethanol embolotherapy. Radiology 170:1059-1066, 1989

29. Yune HY, O'Connor KW, Klatte EC, Olson EW, Becker GJ, Strickler SA: Ethanol thrombo therapy of esophageal varices: Further experience. AJR Am J Roentgenol 144:1049-1053, 1985

30. Zingrillo M, Collura D, Ghiggi MR, Nirchio V, Trischitta V: Treatment of large cold benign thyroid nodules not eligible for surgery with percutaneous ethanol injection. J Clin Endocrinol Metab 83:39053907,1998 